\title{
Ventricular Hypertrophy by ECG Finding
}

National Cancer Institute

\section{Source}

National Cancer Institute. Ventricular Hypertrophy by ECG Finding. NCI Thesaurus. Code C71084.

An electrocardiographic finding suggestive of an enlarg ed cardiac ventricle. 